\title{
Genetic deletion of Urocortin 3 does not prevent functional maturation of beta cells
}

\author{
Jessica L Huang ${ }^{1}$, Sharon Lee', Pelle Hoek¹, Talitha van der Meulen'1, Richard Van ${ }^{1}$ and Mark O Huising ${ }^{1,2}$ \\ 1Department of Neurobiology, Physiology \& Behavior, College of Biological Sciences, University of California, Davis, California, USA \\ 2Department of Physiology and Membrane Biology, School of Medicine, University of California, Davis, California, USA
}

Correspondence should be addressed to M O Huising: mhuising@ucdavis.edu

\begin{abstract}
There is great interest in generating functionally mature beta cells from stem cells, as loss of functional beta cell mass contributes to the pathophysiology of diabetes. Identifying markers of beta cell maturity is therefore very helpful for distinguishing stem cells that have been successfully differentiated into fully mature beta cells from stem cells that did not. Urocortin 3 (UCN3) is a peptide hormone whose expression is associated with the acquisition of functional maturity in beta cells. The onset of its expression occurs after other beta cell maturity markers are already expressed and its loss marks the beginning of beta cell dedifferentiation. Its expression pattern is therefore tightly correlated with beta cell maturity. While this makes UCN3 an excellent marker of beta cell maturity, it is not established whether UCN3 is required for beta cell maturation. Here, we compared gene expression and function of beta cells from Ucn3null mice relative to WT mice to determine whether beta cells are functionally mature in the absence of UCN3. Our results show that genetic deletion of Ucn3 does not cause a loss of beta cell maturity or an increase in beta cell dedifferentiation. Furthermore, virgin beta cells, first identified as insulin-expressing, UCN3-negative beta cells, can still be detected at the islet periphery in Ucn3-null mice. Beta cells from Ucn3-null mice also exhibit normal calcium response when exposed to high glucose. Collectively, these observations indicate that UCN3 is an excellent mature beta cell marker that is nevertheless not necessary for beta cell maturation.
\end{abstract}

\section{Introduction}

Beta cells are the sole source of insulin in the body. Thus, there is much interest in finding ways to generate new beta cells. One of the current methods of regenerating beta cells is differentiating stem cells in vitro (Pagliuca et al. 2014, Rezania et al. 2014, Russ et al. 2015, Nair et al. 2019, Velazco-Cruz et al. 2019, Veres et al. 2019). However, although much progress has been made toward generating beta cells from stem cells, clinically meaningful generation of functionally mature beta cells in vitro has yet to be achieved. Markers that can guide the maturation of beta cells in vitro are therefore of great utility.

The maturation of beta cells in vivo is driven by several key transcription factors such as PDX1, NKX6-1 and MAFA, among others. In mice, insulin-expressing cells first appear around E9.5, with a second, larger wave appearing around E13.5, which corresponds to the onset of MAFA expression (Artner et al. 2010). However, most beta cells do not express MAFA at this early age and remain largely 
functionally immature. Additional markers, such as the peptide hormone Urocortin 3 (UCN3), have emerged as late beta cell maturity markers in recent years.

UCN3 is a member of the corticotropin-releasing factor (CRF) family that selectively binds the G-protein coupled receptor CRFR2 (Lewis et al. 2001). Shortly after its discovery, it was found to be expressed in beta cells ( $\mathrm{Li}$ et al. 2003). UCN3 is co-released with insulin under high glucose conditions and stimulates somatostatin secretion from delta cells, which are the primary cells within the islet that express CRFR2 (van der Meulen et al. 2015). This creates a negative feedback loop that leads to the repression of insulin.

During development, UCN3 is first expressed by some beta cells in the later stages of mouse embryonic development at E17.5 and is not expressed by the majority of beta cells until 2 weeks after birth (Blum et al. 2012, van der Meulen et al. 2012). The late onset of UCN3 expression in beta cells places it well after that of commonly used maturity markers such as MAFA and PDX1 (van der Meulen \& Huising 2014, Hunter \& Stein 2017). This makes UCN3 a useful marker to distinguish between immature insulinpositive cells and fully functional, stem cell-derived beta cells. Furthermore, the expression of UCN3 coincides with the acquisition of functional maturity by mouse beta cells that is marked by a notable uptick in blood glucose values (Blum et al. 2012). Indeed, prematurely inducing the expression of UCN3 in beta cells of mice under 2 weeks of age directly leads to a premature increase in blood glucose levels (van der Meulen et al. 2015). This demonstrates that UCN3 expression is sufficient to trigger the increase in blood glucose levels that, thus, sets the higher blood glucose set point that is associated with the acquisition of functional beta cell maturity.

More recently, we identified a novel sub-population of 'virgin' beta cells specifically located at the periphery of the islet that was discovered by virtue of their lack of UCN3 expression (van der Meulen et al. 2017). These beta cells express insulin, but do not express beta cell maturity markers, including UCN3, MAFA, ERO1LB, G6PC2 and GLUT2. These virgin beta cells are functionally immature and unable to sense glucose, in part, because of their lack of cell-surface expression of GLUT2. On the other side of the spectrum, loss of UCN3 has also been established as a hallmark of beta cell dedifferentiation in diabetes (Blum et al. 2014, van der Meulen et al. 2015). In leptin-deficient $o b / o b$ mice, UCN3 expression is initially indistinguishable from controls until the onset of diabetes, at which point UCN3 expression markedly decreases, which continues as diabetes progresses. The return of UCN3 expression has thus been used to demonstrate re-differentiation of beta cells (Blum et al. 2014). More recently, UCN3 expression has also been reported to be decreased in senescent beta cells in type 1 diabetes (Thompson et al. 2019).

The fact that UCN3 expression tracks closely with beta cell function across different beta cell states raises the question if UCN3 is merely a marker of mature beta cells or whether beta cells directly require UCN3 for their maturation and normal function. Although the receptor for UCN3 is selectively expressed on delta cells and thus cannot affect beta cell maturity in a cell-autonomous or autocrine fashion, UCN3 could indirectly affect beta cell maturity via paracrine, somatostatin-mediated crosstalk within the islet, analogous to its effects on insulin secretion (van der Meulen et al. 2015). Indeed, given the strong correlation of UCN3 with beta cell maturity, the question of causation is regularly raised. To investigate this question, we used genetic ablation of Ucn3 to demonstrate whether beta cells can reach functional maturity in the absence of UCN3.

\section{Materials and methods}

\section{Animals}

Mice were maintained in group housing on a $12 \mathrm{~h}$ light:12 $\mathrm{h}$ darkness cycle with free access to water and standard rodent chow. Ucn3-null mice were described previously (Li et al. 2007). For all experiments, mice heterozygous for the Ucn3-null allele (Ucn3+/, referred to as Ucn3-het) were used to produce knockout mice (Ucn $3^{--}$, referred to as $U c n 3$-null) and control littermates $\left(U c n 3^{+/+}\right.$, referred to as Ucn3-wt, or Ucn3-het). Ucn3-het mice were crossed to mice expressing $T g(I n s 1-c r e / E R T) 1 L p h$, henceforth referred to as MIP-Cre/ERT (Wicksteed et al. 2010), and Gt(ROSA)26Sortm96(CAG-GCaMP6s)Hze, henceforth referred to as lsl-GCaMP6s (Madisen et al. 2015), to produce Ucn3het mice that express either MIP-Cre/ERT or lsl-GCaMP6s. They were then crossed together to produce Ucn3-null mice hemizygous for both MIP-Cre/ERT and lsl-GCaMP6s. These mice selectively and efficiently expressed GCaMP6s in beta cells upon oral administration of tamoxifen (Sigma-Aldritch, T5648) dissolved in sunflower seed oil (Trader Joe's, Monrovia, CA, USA) at $20 \mathrm{mg} / \mathrm{mL}$ for 5 consecutive days. Killing and islet isolation were carried out after a 3-day washout period. Heterozygous B6.Cg-Lep ${ }^{o b} / \mathrm{J}$ breeders were bought from The Jackson Laboratories to produce homozygous mice, referred to as ob/ob (Coleman 1978). 
Mice used were between 4 and 6 months of age unless otherwise indicated. All mouse experiments were approved by the UC Davis Institutional Animals Care and Use Committee and were performed in compliance with the Animal Welfare Act and the Institute for Laboratory Animal Research (ILAR) Guide to the Care and Use of Laboratory Animals.

\section{Glucose tolerance test}

Mice were weighed after an overnight fast, then given a bolus of $2 \mathrm{~g} / \mathrm{kg}$ glucose (dextrose; Sigma-Aldritch, D9559) via i.p. injection. Blood glucose levels were collected over a 2-h time period using tail vein blood by the OneTouch Ultra glucometer.

\section{Immunofluorescence}

Immunofluorescence was conducted as follows: slides were washed for $5 \mathrm{~min}$ three times in KPBS, then incubated with antibodies diluted in KPBS supplemented with $2 \%$ donkey serum and $0.4 \%$ Triton X-100 overnight at $4^{\circ} \mathrm{C}$. Slides were then washed three more times in KPBS, incubated with secondary antibodies and DAPI $(1 \mu \mathrm{g} / \mathrm{mL})$ diluted in donkey block for $45 \mathrm{~min}$ at room temperature and washed three more times before embedding in Prolong Gold Antifade (Thermo Fisher Scientific). Images were obtained on a Nikon A1R+ confocal microscope and Ucn3-wt and Ucn3-het were used as controls to compare to Ucn3-null tissues.

\section{Islet isolation}

Islets were isolated by injecting $2 \mathrm{~mL}$ collagenaseP (0.8 $\mathrm{mg} / \mathrm{mL}$, Invitrogen) dissolved in HBSS (Roche Diagnostics) into the pancreas via the common bile duct while the ampulla of Vater was clamped. The pancreas was then collected and submerged with an additional $2 \mathrm{~mL}$ of collagenase solution, incubated at $37^{\circ} \mathrm{C}$ for $13 \mathrm{~min}$ and then dissociated through gentle manual shaking. The dissociated pancreas was then washed three times with cold HBSS containing 5\% NCS, then passed through a nylon mesh (pore size $425 \mu \mathrm{m}$, Small Parts) and isolated by density gradient centrifugation on a Histopaque gradient (1.077 g/mL, Sigma) for $20 \mathrm{~min}$ at $1400 \boldsymbol{g}$ without brake. Islets were collected from the interface, washed once with cold HBSS containing 5\% NCS and then handpicked several times under a dissecting microscope before culturing in RPMI (Roche Diagnostics) containing $5.5 \mathrm{mM}$ glucose, 10\% FBS and pen/strep.

\section{Next generation sequencing and bioinformatics}

RNA isolation, library preparation, sequencing and alignment were performed as previously described (van der Meulen et al. 2015). Briefly, RNA was isolated from Trizol by a chloroform extraction, then precipitated by isopropanol and cleaned up over an RNEasy microcolumn (Qiagen) per the manufacturer's instructions. RNA quality was determined by BioAnalyzer (Agilent). Libraries were generated using the TruSeq RNA Sample Prep Kit v2 (Illumina Inc., San Diego, CA, USA) and sequenced at 50 cycles, single read on an Illumina HiSeq 2000 platform. Reads were aligned to mouse genome version $\mathrm{mm} 9$ using STAR.

\section{Western blotting}

A50 islets were lysed using $30 \mu \mathrm{L}$ sample treatment buffer (50 mM Tris ( $\mathrm{pH} \mathrm{7.5),} 100 \mathrm{mM}$ dithiothreitol, $2 \%$ (weight/volume) sodium dodecyl sulfate, $0.1 \%$ (weight/volume) bromophenol blue and 10\% (weight/ volume) glycerol). Lysates were run on a SDS/PAGE gel and transferred nitrocellulose membranes (Whatman, GE). Immunoblotting was done using rabbit anti-UCN3 (\#7218) (gift from Dr Wylie Vale, 1:1000) and the secondary antibody donkey anti-rabbit conjugated to HRP (GE Healthcare U.K. Ltd., 1:5000).

\section{Functional imaging}

After isolation, islets were placed in 35-mm dishes with a glass bottom (\#1.5, MatTek Corporation, Ashland, MA, USA) and allowed to adhere by culturing overnight. Islets were imaged over time in $3 \mathrm{D}$ on a Nikon A1R+ confocal microscope using a 20x lens. Continuous perfusion of islets with Krebs Ringer Buffer was done using a Masterflex peristaltic pump at $2.5 \mathrm{~mL}$ per minute. Islets were perfused with low glucose for $10 \mathrm{~min}$ to obtain baseline fluorescence, then switched to high glucose at the indicated time point. Regions of interest were drawn around single cells or single islets to obtain fluorescence intensity using Nikon Elements software.

\section{Antibodies}

Primary antibodies used were chicken polyclonal antiinsulin (Abcam ab14042, 1:1000), rat monoclonal antiinsulin (R\&D Systems, 1:500), guinea pig polyclonal anti-insulin (Dako \#A0564; 1:500), rabbit (\#7218) or guinea pig (\#044) polyclonal anti-UCN3 
(gift from Dr Wylie Vale, 1:1000), rabbit polyclonal anti-MAFA (Bethyl Laboratories, Montgomery, TX, USA; IHC-00352, 1:100), rabbit polyclonal anti-MAFB (Bethyl Laboratories IHC-00351, 1:100), rabbit polyclonal anti-PDX1 (Abcam ab47267, 1:500), rabbit polyclonal anti-ERO1LB (gift from Dr David Ron, 1:300), rabbit polyclonal anti-G6PC2 (Gift from Drs Jay Walters and Howard Davidson, 1:200), rabbit polyclonal anti-GLUT2 (EMD Millipore \#07-1402I, 1:1000) and rabbit polyclonal anti-ALDH1A3 (Novus Biologicals NBP2-15339, 1:500). Secondary antibodies were obtained from Jackson ImmunoResearch and used at a 1:600 dilution.

\section{Statistical analysis}

Data were analyzed by Student's t-test, corrected for multiple comparisons using the Holm-Sidak method where appropriate and represented as mean \pm s.E.M., with $n$ representing number of animals in each group. Differences were considered significant when $P$ value was $<0.05$. Statistics were computed using Prism (GraphPad Software).

\section{Results}

\section{Expression of beta cell maturity genes persists in Ucn3-null mice}

We first evaluated our previously published whole islet transcriptomes of Ucn3-null mice vs Ucn3-wt littermates (van der Meulen et al. 2015). Several genes are differentially regulated between Ucn3-wt and Ucn3-null mice, with 22 genes enriched in Ucn3-wt mice and 7 genes enriched in Ucn3-null mice (Fig. 1A, Supplementary Fig. 1A and Supplementary Table 1 , see section on supplementary materials given at the end of this article). Ucn3-null islets demonstrate a loss of UCN3, confirming successful knockout of Ucn3, with no observable compensation in UCN and UCN2, which are barely detectable if at all in both Ucn3-wt and Ucn3-null mice (Supplementary Fig. 1B and C) and do not change in Ucn3-null mice (Fig. 1A). Expression of CRHR2, the receptor for UCN3, is unchanged in Ucn3-null mice (Supplementary Fig. 1D). Of the 22 genes with lower expression in Ucn3-null mice, several are known delta cell markers such as Sst, Hhex and Rbp4, which was previously reported and caused by a significant reduction in delta cells in the absence of UCN3 (van der Meulen et al. 2015). However, there was no significant difference in the expression of Ins 1 or Ins 2 expression in Ucn3-null mice relative to their WT littermates (Fig. 1B).
To further confirm that there is no difference in beta cell maturity at the transcriptional level, we examined the gene expression of several established beta cell maturity markers, including MafA, Pdx1, Ero1lb, G6pc2 and Slc2a2.
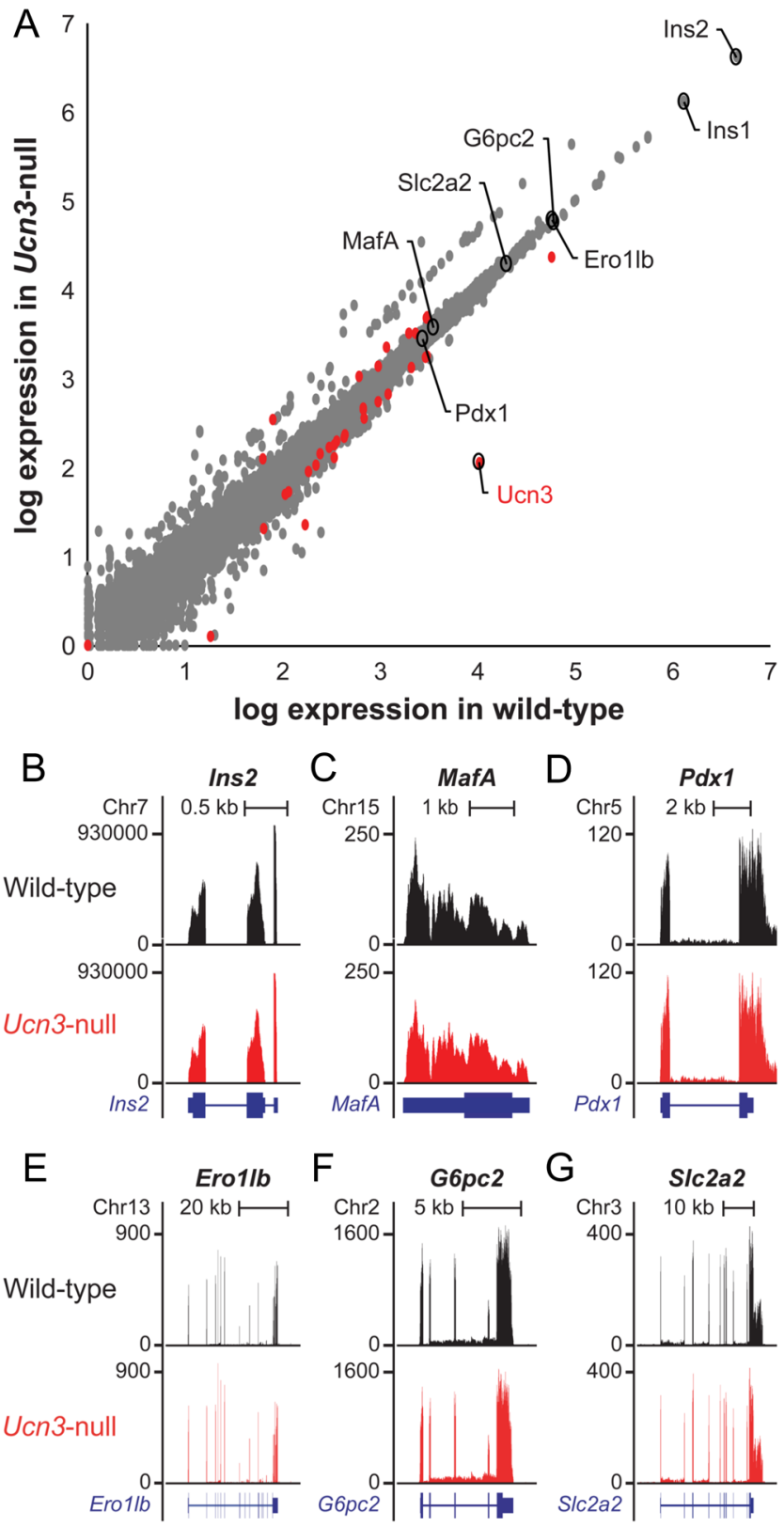

\section{Figure 1}

Genes encoding beta cell maturity markers are not differentially regulated between Ucn3-null mice and WT littermates. (A) Volcano plot showing global gene expression in WT vs Ucn3-null mice. Differentially expressed genes are denoted as red circles. Select markers of beta cell maturity are circled and labeled. Ucn3 is depleted in Ucn3-null mice, confirming knockout, while other beta cell maturity markers are not affected at the transcript level. (B, C, D, E, F and G) Visualization of relative reads of different transcripts in Ucn3-null mice (red) compared to WT (black). (B) Ins2, (C) MafA, (D) Pdx1, (E) Ero1/b, (F) G6pc2 and (G) S/c2a2. 
All of these were expressed at similar levels between Ucn3null and WT mice (Fig. 1C, D, E, F and G).

Western blotting and immunofluorescence by UCN3 antiserum confirmed that UCN3 is no longer expressed in Ucn3-null mice but is retained in both Ucn3-wt and Ucn3-het mice (Fig. 2A and B). The transcription factor MAFA, which is required for mature beta cell identity, is expressed in the nuclei of most beta cells in both WT and Ucn3-null mice (Fig. 2C). Similarly, PDX1 is expressed in the nucleus of beta cells as well (Fig. 2D). ERO1LB and
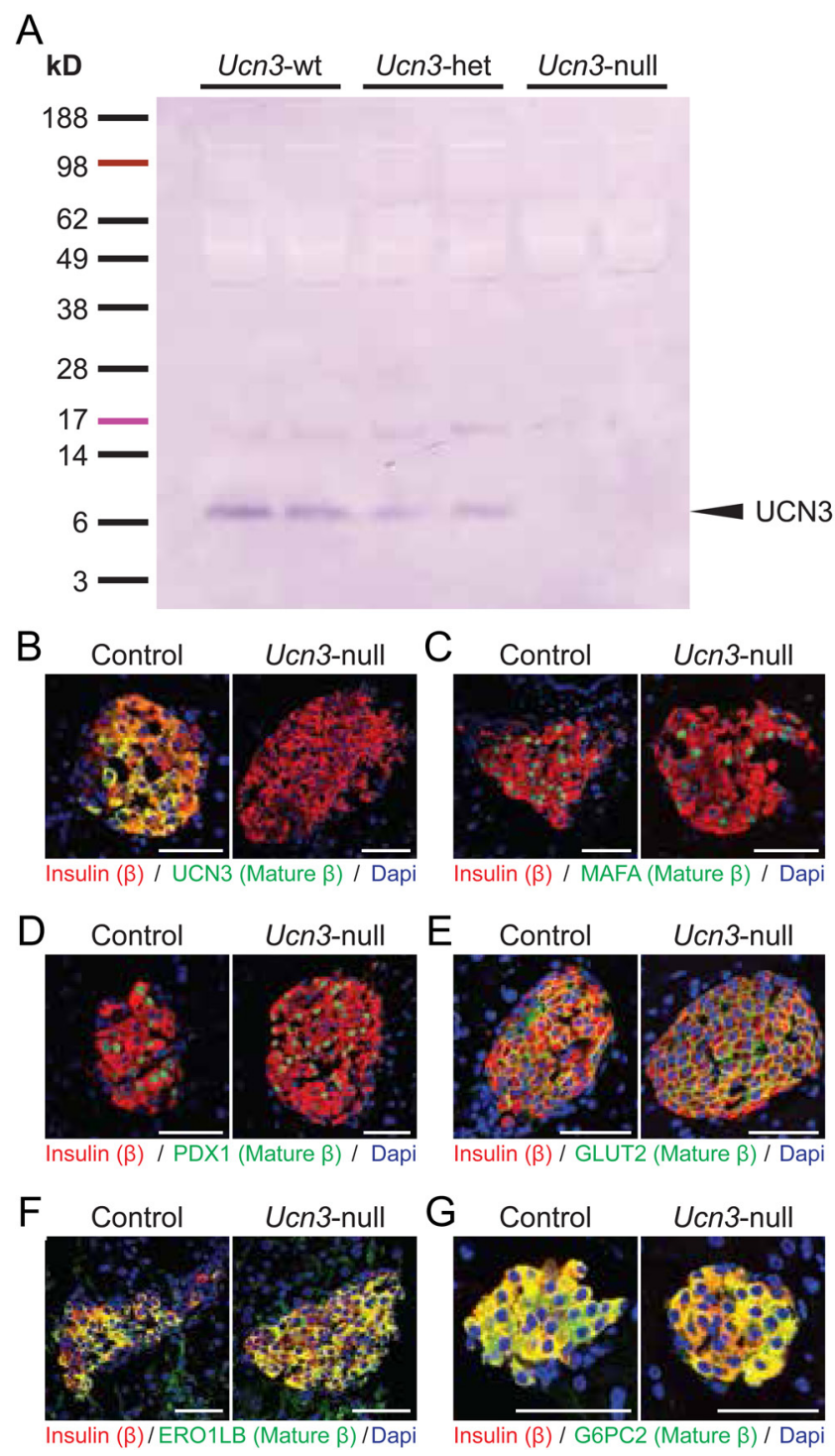

Figure 2

UCN3 is not necessary for beta cells to express maturity markers. (A and B) Western blot and immunofluorescence confirm the loss of UCN3 in Ucn3-null mice. (B, C, D, E, F and G) Co-localization of insulin and select beta cell maturity markers in islets of Ucn3-null mice and littermate controls. Insulin is stained in red, the marker of interest is in green and nuclei stained by DAPI are in blue. (B) UCN3, (C) MAFA, (D) PDX1, (E) ERO1LB, (F) G6PC2 and (G) GLUT2. Scale bars indicate $50 \mu \mathrm{m}$.

(C) 2020 Society for Endocrinology Published by Bioscientifica Ltd. Printed in Great Britain
G6PC2 both have strong overlap with insulin in both WT and Ucn3-null mice (Fig. 2E, F and Supplementary Fig. 2). Immunoreactivity of the glucose transporter GLUT2, encoded by Slc2a2, is detected on the surface of beta cells in both Ucn3-null and control mice (Fig. 2G). Overall, these results indicate that the expression of a panel of established beta cell maturity markers is unperturbed in the absence of UCN3.

\section{Beta cell dedifferentiation is not increased in Ucn3-null mice}

The preservation of the expression of beta cell maturity markers in Ucn3-null compared to WT mice established that UCN3 expression is not necessary for beta cell maturation. Conversely, the loss of UCN3 expression in beta cells is also a sensitive and early marker of beta cell dedifferentiation (Blum et al. 2014, van der Meulen et al. 2015). To investigate if the genetic deletion of UCN3 increases beta cell dedifferentiation, we assessed the expression of several beta cell dedifferentiation markers. Aldehyde dehydrogenase 1 family member A3 (ALDH1A3) is an established marker of dedifferentiated beta cells (Kim-Muller et al. 2016). As expected, ALDH1A3 was absent from the majority of beta cells in lean, WT mice at $0.28 \% \pm 0.24 \%$ (Fig. 3). However, the genetic deletion of Ucn3 in Ucn3-null mice by itself did not cause a significant increase in the number of ALDH1A3expressing beta cells $(0.50 \% \pm 0.43 \%)$. In contrast, beta cells in $o b / o b$ mice did express ALDH1A3 in almost half of all beta cells $(46.70 \% \pm 1.93 \%)$, consistent with published observations (Kim-Muller et al. 2016), while UCN3 was no longer detectable in a similarly sized proportion of beta cells in islets from $o b / o b$ mice $(50.17 \% \pm 34.63 \%)$ (Fig. 3A, 3B). Other dedifferentiation markers such as neurogenin-3 (Neurog3) and vimentin (Vim) also did not change significantly in Ucn3-null islets (Supplementary Fig. 1E and F). Collectively, this suggests that the loss of UCN3 by itself does not cause beta cell dedifferentiation as measured by the expression of ALDH1A3, Neurog3 and Vim.

\section{Virgin beta cells remain identifiable in Ucn3-null mice}

Recently, our lab discovered virgin beta cells as a novel beta cell type that is found at the periphery of the mouse islet and is recognized by its lack of UCN3, as well as other beta cell maturity markers. As we originally identified virgin beta cells based on their absence of UCN3 expression, we wanted to determine whether they 

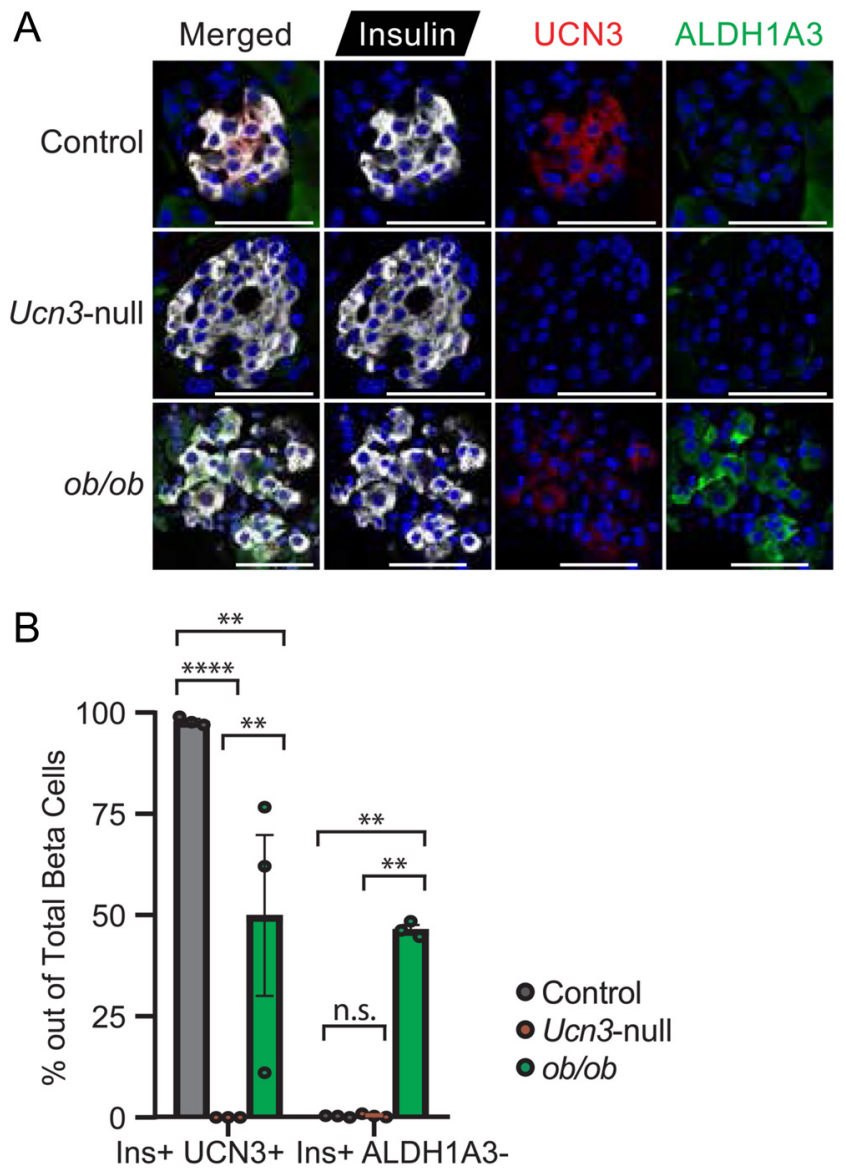

\section{Figure 3}

The absence of UCN3 does not lead to beta cell dedifferentiation. (A) Immunofluorescence of insulin, UCN3 and ALDH1A3 in islets from WT, Ucn3-null and ob/ob mice. Control mice co-express insulin and UCN3 in beta cells, but express little to no ALDH1A3. In contrast, more insulinpositive beta cells in ob/ob islets express ALDH1A3 and exhibit weaker immunoreactivity of UCN3. Ucn3-null mice, like their control littermates, express little to no ALDH1A3 in beta cells but lack expression of UCN3. Scale bars indicate $50 \mu \mathrm{m}$. (B) Quantification of insulin-positive, UCN3positive and insulin-positive, ALDH1A3-positive cells in control, Ucn3-null and ob/ob mice, as percentage of total beta cells. $n=3$ animals per group, at least 10 islets and 500 cells counted per animal. Error bars reflect S.E.M. $* * P<0.01, * * * * P<0.001$.

remained detectable upon genetic ablation of Ucn3 and if they maintained their characteristic location at the periphery of the islet. Staining for insulin, UCN3 and GLUT2 readily revealed virgin beta cells in control mice expressing UCN3, most of which also lacked cell-surface GLUT2 (csGLUT2) expression as previously shown (van der Meulen et al. 2017) (Fig. 4A). Insulin-expressing cells lacking csGLUT2 expression was also found in Ucn3-null mice (Fig. 4B). While the fraction of insulin+ UCN3- virgin beta cells is similar to what we have previously established $(1.21 \% \pm 0.81 \%)$, the fraction of insulin+ csGLUT2- beta cells is higher $(3.13 \% \pm 1.27 \%$ in control mice, $2.96 \% \pm 0.32 \%$ in Ucn3-null mice) (Fig. 4C), similar to what we previously published (van der Meulen et al. 2017). In control mice, this is due to the presence of several mature insulin+ UCN3+ cells without csGLUT2 expression. As the fraction of insulin+ csGLUT2- is similar in the Ucn3-null mice, it is likely that these cells similarly represent both virgin and mature beta cells. Nevertheless, we conclude that many of the insulin+ csGLUT2- cell identified in the Ucn3-null mice are virgin beta cells, as they remain at their characteristic location of the islet periphery. We also looked at G6PC2 expression as another marker (Fig. 4D and E). Here the data also show the expected fraction of insulin+ UCN3cells $(1.46 \% \pm 0.25 \%)$, with fewer insulin+ G6PC2- cells $(0.50 \% \pm 0.16 \%)$ (Fig. $4 \mathrm{~F})$. These numbers are in agreement with our previously published data (van der Meulen et al. 2017). The fraction of insulin+ G6PC2- was not significantly different in Ucn3-null mice $(0.49 \% \pm 0.21 \%)$. All insulin+ UCN3- and insulin+ G6PC2- cells localize to the islet periphery in both Ucn3-null mice and littermate controls. The presence of MAFB, which is absent from mature beta cells but enriched among peripheral virgin beta cells (Supplementary Fig. 3), further supports the continued presence of virgin beta cells despite the deletion of the Ucn3 gene.

\section{Beta cells in Ucn3-null mice exhibit normal calcium response to glucose}

We established that beta cells maintain the expression of beta cell maturity markers despite the absence of UCN3. This implies that these beta cells remained functionally mature. To interrogate the functional maturity of beta cells directly, we crossed Ucn3-null mice to mice that express the calcium reporter GCaMP6s (Madisen et al. 2015) specifically in beta cells using MIP-Cre/ERT (Wicksteed et al. 2010). Mindful of the potential confounds of the MIP-Cre/ERT mouse line (Carboneau et al. 2016), we used mice that were WT for Ucn3 but transgenic for $M I P-C r e / E R T$ as controls, thus eliminating any confounds owing to the differential presence of MIP-Cre/ERT. This strategy enabled the resolution of the kinetics of beta cell calcium response, which closely match the kinetics of insulin secretion (Bergsten et al. 1994), at single-cell resolution. Upon stimulation by high glucose, beta cells from both Ucn3-null mice and their WT controls respond with an increase in intracellular calcium, as represented by an increase in fluorescence (Fig. 5). Although there is islet to islet variation in calcium response, beta cells within the same islet all respond in unison. The calcium response is also pulsatile in both Ucn3-null and WT mice, 


\begin{tabular}{|l|l|l|r|r|}
$\begin{array}{l}\text { Journal of } \\
\text { Endocrinology }\end{array}$ & J L Huang et al. & $\begin{array}{l}\text { UCN3 marks but does not drive } \\
\beta \text { cell maturity }\end{array}$ & $\mathbf{2 4 6 : 1}$ & $\mathbf{7 5}$ \\
\hline
\end{tabular}

A

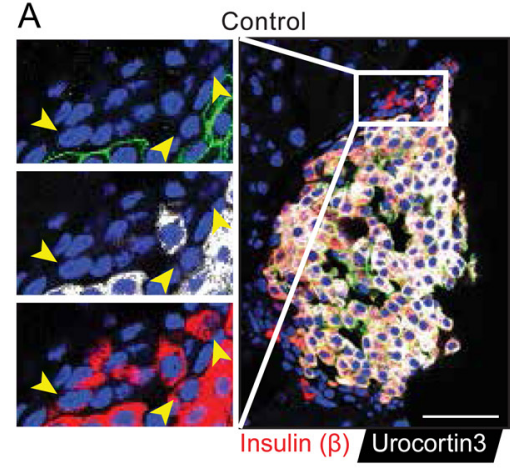

D
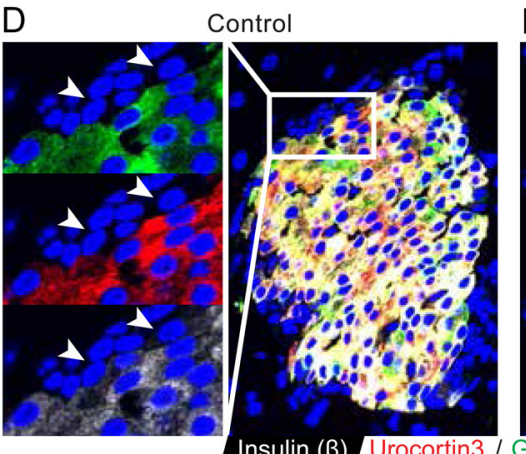

B

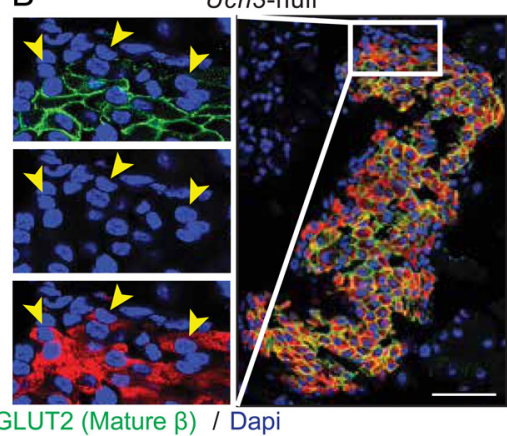

Control
E

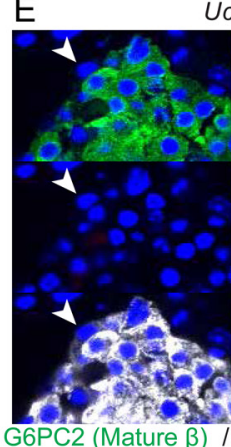

Ucn3-null

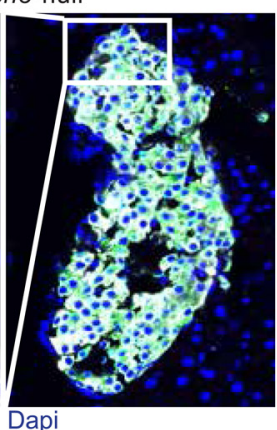

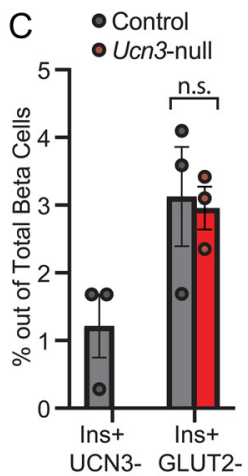

$\mathrm{F}$

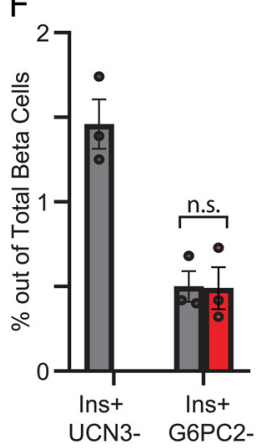

Figure 4

Virgin beta cells are maintained at the islet periphery in the absence of UCN3. (A and B) Immunofluorescence of insulin, UCN3 and GLUT2 in islets from (A) control and (B) Ucn3-null mice. Insulin and UCN3 co-localize in beta cells, while GLUT2 is expressed on the membrane of the beta cells. Arrows point to virgin beta cells, which are insulin-positive but UCN3-negative and GLUT2-negative in control mice and insulin-positive but GLUT2-negative in Ucn3-null mice. Scale bars indicate $50 \mu \mathrm{m}$. (C) Quantification of insulin-positive, UCN3-negative cells in control mice and insulin-positive, csGLUT2-negative cells in control and Ucn3-null mice, as percentage of total beta cells. (D and E) Immunofluorescence of insulin, UCN3 and G6PC2 in islets from (A) control and (B) Ucn3-null mice. Insulin, UCN3 and G6PC2 are co-expressed in the majority of beta cells in control mice. Arrows point to virgin beta cells that are insulin-positive but UCN3- and G6PC2-negative. (F) Quantification of insulin-positive, UCN3-negative cells in control mice and insulin-positive, G6PC2negative cells in control and Ucn3-null mice. For both C and F, there is no quantification for insulin+ UCN3- cells in the Ucn3-null mice as all cells are UCN3- and thus not representative of the virgin beta cell population. $n=3$ animals per group, at least 10 islets and 500 cells counted per animal. Error bars reflect S.E.M.

with the first phase response exhibiting a longer duration than the remaining pulses. This confirms that beta cells within each islet from Ucn3-null mice remain capable of sensing and responding to high glucose in a pulsatile and synchronized manner.

\section{Discussion}

The peptide hormone UCN3 is one of the last hormones to appear during the postnatal maturation of beta cells (Blum et al. 2012, van der Meulen et al. 2012). The onset of UCN3 expression trails expression of other established beta cell maturity markers such as MAFA (van der Meulen \& Huising 2014), coincides with the acquisition of functional maturity in beta cells (Blum et al. 2012) and has been reported to be expressed in functional beta cells derived from transplanted stem cells that were allowed to mature over time in vivo (van der Meulen et al. 2012, Xie et al. 2013). While these observations have helped to establish UCN3 as a very useful marker to identify functionally mature beta cells, both in the pancreas and in differentiated stem cells, the question of whether UCN3 merely marks mature beta cells or is required for beta cells to mature comes up regularly. Here, we resolve this question by demonstrating that genetic deletion of Ucn3 does not lead to loss of other beta cell maturity markers, nor does it increase the level of expression of beta cell dedifferentiation markers, as determined by RNAsequencing and validated by immunofluorescence. These observations indicate that beta cells continue to express other maturity markers upon Ucn 3 deletion. This suggests that UCN3 is a maturity marker, but may not directly act on the beta cell to promote beta cell maturation. This is in agreement with reports that treatment of fetal beta cells with UCN3 was insufficient to accelerate beta cell 
A Wild-type Beta Cell Calcium Response

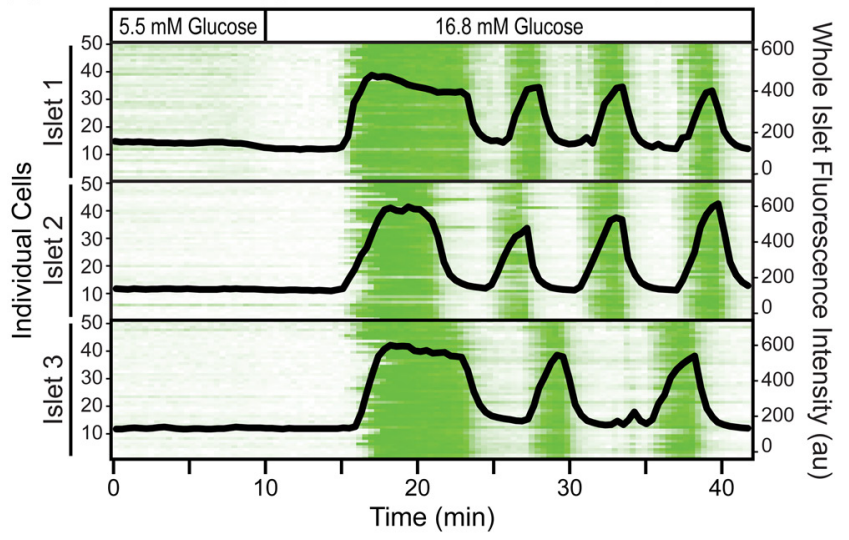

B Ucn3-null Beta Cell Calcium Response

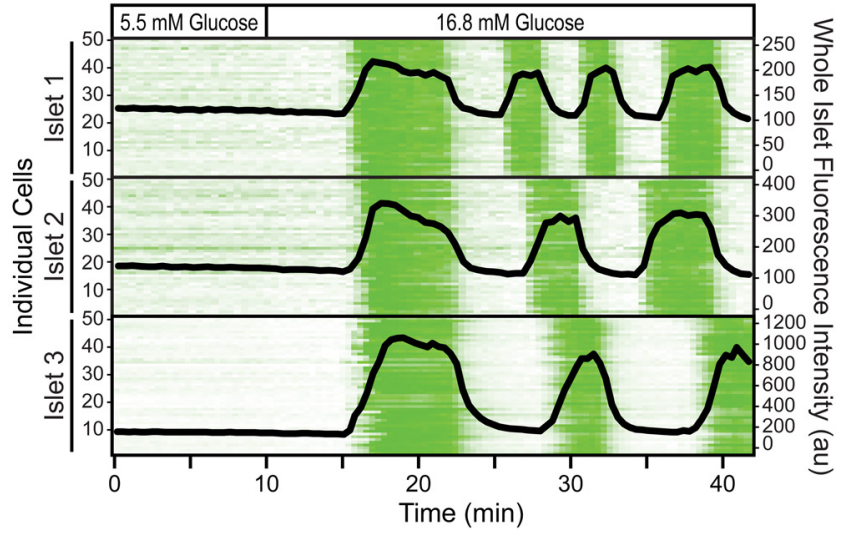

\section{Figure 5}

Beta cell calcium response remains unaltered in mice that do not express UCN3. Calcium responses of several intact islets were obtained simultaneously over time. All islets expressed the calcium sensor GCaMP6s selectively in beta cells (MIP-Cre/ERT $\times$ Isl-GCaMP6s) with or without Ucn3-null in the background. The trace began with low glucose and was switched to high glucose at the indicated timepoint. The calcium traces of beta cells within three representative islets from (A) WT mice and (B) Ucn3-null mice are shown as intensity plots. Each line represents the activity of an individual beta cell over time, and green represents an increase in fluorescence intensity. A line graph of the fluorescence intensity at the whole islet level is overlaid over each intensity plot.

maturation (Blum et al. 2012, Liu \& Hebrok 2017). This also agrees closely with the fact that the UCN3 receptor CRHR2 $\alpha$ is not expressed in beta cells, but is instead selectively expressed by delta cells (DiGruccio et al. 2016), which respond to UCN3 with increased somatostatin release (van der Meulen et al. 2015).

Furthermore, beta cells from Ucn3-null mice exhibit synchronized, pulsatile calcium responses to high glucose comparable to the responses of beta cells from WT mice. The rise in intracellular calcium is required for the mobilization of insulin secretory granules, and synchronized, oscillatory calcium response has been demonstrated to be critical for the secretion of insulin (Rorsman \& Renström 2003). Our data therefore suggests that beta cells in Ucn3-null mice retain proper insulin secretion capabilities. However, while the kinetics of beta cell calcium responses are similar between WT and Ucn3-null mice, GCaMP6s is not calibrated to detect absolute calcium concentrations and instead detects relative changes in intracellular calcium. These are known to track the kinetics (Bergsten et al. 1994, Ravier et al.2002), but not necessarily the magnitude of insulin secretion. Furthermore, increases in intracellular calcium levels may not represent a proportional increase in secretion. Indeed, we have previously demonstrated that beta cells in Ucn3-null mice exhibit exaggerated first- and secondphase insulin secretion that can be normalized acutely by addition of synthetic UCN3 peptide (van der Meulen et al. 2015). These mice also display increased glucose tolerance (van der Meulen et al. 2015) (Supplementary Fig. 4), setting up the interesting paradox that UCN3 is a marker for functional maturity that restrains glucosestimulated insulin secretion. We have proposed that such feedback inhibition maintained by UCN3-dependent, somatostatin-mediated restraint on insulin secretion prevents reactive hypoglycemia (Huising et al. 2018).

We also demonstrate that beta cell states that are normally marked by the absence of UCN3, such as immature 'virgin' beta cells, can still be detected despite the genetic ablation of the Ucn 3 gene. Virgin beta cells were still readily identifiable at the islet periphery using alternative maturity markers such as csGLUT2 instead of UCN3 (Beamish et al. 2016). Conversely, the loss of UCN3 expression is an early hallmark of beta cell dedifferentiation (Blum et al. 2014, van der Meulen et al. 2015) that coincides with an increase in the expression of the dedifferentiation marker ALDH1A3 (Kim-Muller et al. 2016). Indeed, we confirmed the increase of ALDH1A3 expression in beta cells from $o b / o b$ mice and demonstrated that there is a corresponding decrease in UCN3 in beta cells. However, the genetic deletion of UCN3 did not by itself cause an increase in ALDH1A3 expression in beta cells. This is similar to how the dedifferentiation marker ALDH1A3 did not induce beta cell dedifferentiation when over expressed (Kim-Muller et al. 2016). Therefore, the expression of ALDH1A3 and the loss of UCN3 expression are both strong markers of dedifferentiation, but do not drive beta cell dedifferentiation. Our experiment establishes that loss of UCN3 expression is in fact an effect of beta cell dedifferentiation that occurs reliably and early on. Interestingly, Ucn3-null mice have been previously reported to be protected from metabolic dysfunction associated with a high-fat diet or old age (Li et al. 2007). 
This is likely due to the decreased somatostatin tone seen in Ucn3-null mice (van der Meulen et al. 2015), which would allow them to maintain increased insulin secretion to compensate for increased peripheral insulin resistance. Indeed, restoration of UCN3 expression in moderately glycemic $o b / o b$ mice at a disease stage where endogenous UCN3 has been downregulated immediately aggravates hyperglycemia (van der Meulen et al. 2015), suggesting that the downregulation in effect is protective under these conditions of increased peripheral insulin resistance.

Collectively, our observations here indicate that, although the onset of the endogenous expression of UCN3 marks the point at which beta cells acquire functional maturity, beta cells will express other common maturity markers and develop the capability for synchronized, pulsatile calcium responses to high glucose despite the lack of UCN3. These observations that UCN3 is not necessary for beta cell maturation should not distract from the utility of endogenous UCN3 as an excellent beta cell maturity marker under circumstances other than the genetic manipulation of the Ucn3 gene. Furthermore, although UCN3 does not directly signal to beta cells, the possibility that the somatostatin secretion triggered by endogenous UCN3 contributes indirectly to mature beta cell identity and function remains. The onset of UCN3 expression around 2 weeks after birth in mice is correlated with a distinct rise in the glycemic set point (Blum et al. 2012), which has long been attributed to the maturation of beta cells (Rozzo et al. 2009, Jermendy et al. 2011). We have previously shown that premature induction of UCN3 specifically in beta cells causes a premature increase in the glycemic set point in mice (van der Meulen et al. 2015). This suggests that UCN3-stimulated somatostatin secretion is the underlying mechanism responsible for the rise in glycemic set point by attenuating insulin secretion, underscoring the importance of paracrine pathways for normal islet function. Whether somatostatin contributes to beta cell maturation beyond this direct attenuation of insulin secretion is an important question that remains unresolved.

\section{Supplementary materials}

This is linked to the online version of the paper at https://doi.org/10.1530/ JOE-19-0535.

\section{Declaration of Interest}

The authors declare that there is no conflict of interest that could be perceived as prejudicing the impartiality of the research reported.

\section{Funding}

This work was supported by the National Institute of Diabetes and Digestive and Kidney Disease (NID DK-110276), the Juvenile Diabetes Research Foundation (CDA-2-2013-54 and 2-SRA-2019-700-S-B) and the American Diabetes Association (\#1-19-IBS-078). J L H was supported by a National Institute of General Medical Sciences-funded Pharmacology Training Program (T32 GM-099608). S L was supported by the NSF Graduate Research Fellowship (1650042), the UC Davis Training Program in Molecular and Cellular Biology (T32 GM-007377) and the UC Davis NSF Bridge to Doctorate Program (1612490).

\section{Acknowledgements}

The authors thank Jerice Banola, Ariana Momen and Mohammad Pourhosseinzadeh for assistance with cell quantifications.

\section{References}

Artner I, Hang Y, Mazur M, Yamamoto T, Guo M, Lindner J, Magnuson MA \& Stein R 2010 MafA and MafB regulate genes critical to $\beta$-cells in a unique temporal manner. Diabetes 59 2530-2539. (https://doi.org/10.2337/db10-0190)

Beamish CA, Strutt BJ, Arany EJ \& Hill DJ 2016 Insulin-positive, Glut2low cells present within mouse pancreas exhibit lineage plasticity and are enriched within extra-islet endocrine cell clusters. Islets 8 65-82. (https://doi.org/10.1080/19382014.2016.1162367)

Bergsten P, Grapengiesser E, Gylfe E, Tengholm A \& Hellman B 1994 Synchronous oscillations of cytoplasmic $\mathrm{Ca} 2+$ and insulin release in glucose-stimulated pancreatic islets. Journal of Biological Chemistry 269 8749-8753.

Blum B, Hrvatin S, Schuetz C, Bonal C, Rezania A \& Melton DA 2012 Functional beta-cell maturation is marked by an increased glucose threshold and by expression of urocortin 3. Nature Biotechnology 30 261-264. (https://doi.org/10.1038/nbt.2141)

Blum B, Roose AN, Barrandon O, Maehr R, Arvanites AC, Davidow LS, Davis JC, Peterson QP, Rubin LL \& Melton DA 2014 Reversal of $\beta$ cell de-differentiation by a small molecule inhibitor of the TGF $\beta$ pathway. eLife 3 e02809. (https://doi.org/10.7554/eLife.02809)

Carboneau BA, Le TDV, Dunn JC \& Gannon M 2016 Unexpected effects of the MIP-CreER transgene and tamoxifen on $\beta$-cell growth in C57Bl6/J male mice. Physiological Reports 4 e12863. (https://doi. org/10.14814/phy2.12863)

Coleman DL 1978 Obese and diabetes: two mutant genes causing diabetes-obesity syndromes in mice. Diabetologia 14 141-148. (https://doi.org/10.1007/BF00429772)

DiGruccio MR, Mawla AM, Donaldson CJ, Noguchi GM, Vaughan J, Cowing-Zitron C, van der Meulen T \& Huising MO 2016 Comprehensive alpha, beta and delta cell transcriptomes reveal that ghrelin selectively activates delta cells and promotes somatostatin release from pancreatic islets. Molecular Metabolism 5 449-458. (https://doi.org/10.1016/j.molmet.2016.04.007)

Huising MO, van der Meulen T, Huang JL, Pourhosseinzadeh MS \& Noguchi GM 2018 The difference $\delta$-cells make in glucose control. Physiology 33 403-411. (https://doi.org/10.1152/physiol.00029.2018)

Hunter CS \& Stein RW 2017 Evidence for loss in identity, de-differentiation, and trans-differentiation of islet $\beta$-cells in type 2 diabetes. Frontiers in Genetics 8 35. (https://doi.org/10.3389/ fgene.2017.00035)

Jermendy A, Toschi E, Aye T, Koh A, Aguayo-Mazzucato C, Sharma A, Weir GC, Sgroi D \& Bonner-Weir S 2011 Rat neonatal beta cells lack the specialised metabolic phenotype of mature beta cells. Diabetologia 54 594-604. (https://doi.org/10.1007/s00125-0102036-x) https://joe.bioscientifica.com

https://doi.org/10.1530/JOE-19-0535 (c) 2020 Society for Endocrinology Published by Bioscientifica Ltd. 
Kim-Muller JY, Fan J, Kim YJR, Lee SA, Ishida E, Blaner WS \& Accili D 2016 Aldehyde dehydrogenase 1 a3 defines a subset of failing pancreatic $\beta$ cells in diabetic mice. Nature Communications 712631. (https://doi.org/10.1038/ncomms12631)

Lewis K, Li C, Perrin MH, Blount A, Kunitake K, Donaldson C, Vaughan J, Reyes TM, Gulyas J, Fischer W, et al. 2001 Identification of urocortin III, an additional member of the corticotropin-releasing factor (CRF) family with high affinity for the CRF2 receptor. PNAS 98 7570-7575. (https://doi.org/10.1073/pnas.121165198)

Li C, Chen P, Vaughan J, Blount A, Chen A, Jamieson PM, Rivier J, Smith MS \& Vale W 2003 Urocortin III is expressed in pancreatic $\beta$-cells and stimulates insulin and glucagon secretion. Endocrinology 144 3216-3224. (https://doi.org/10.1210/en.2002-0087)

Li C, Chen P, Vaughan J, Lee KF \& Vale W 2007 Urocortin 3 regulates glucose-stimulated insulin secretion and energy homeostasis. PNAS 104 4206-4211. (https://doi.org/10.1073/pnas.0611641104)

Liu JSE \& Hebrok M 2017 All mixed up: defining roles for $\beta$-cell subtypes in mature islets. Genes and Development 31 228-240. (https://doi. org/10.1101/gad.294389.116)

Madisen L, Garner AR, Shimaoka D, Chuong AS, Klapoetke NC, Li L, van der Bourg A, Niino Y, Egolf L, Monetti C, et al. 2015 Transgenic mice for intersectional targeting of neural sensors and effectors with high specificity and performance. Neuron 85 942-958. (https://doi. org/10.1016/j.neuron.2015.02.022)

Nair GG, Liu JS, Russ HA, Tran S, Saxton MS, Chen R, Juang C, Li ML, Nguyen VQ, Giacometti S, et al. 2019 Recapitulating endocrine cell clustering in culture promotes maturation of human stem-cell-derived $\beta$ cells. Nature Cell Biology 21 263-274. (https://doi.org/10.1038/ s41556-018-0271-4)

Pagliuca FW, Millman JR, Gürtler M, Segel M, Van Dervort A, Ryu JH, Peterson QP, Greiner D \& Melton DA 2014 Generation of functional human pancreatic $\beta$ cells in vitro. Cell 159 428-439. (https://doi. org/10.1016/j.cell.2014.09.040)

Ravier MA, Sehlin J \& Henquin JC 2002 Disorganization of cytoplasmic $\mathrm{Ca} 2+$ oscillations and pulsatile insulin secretion in islets from ob/ob mice. Diabetologia 45 1154-1163. (https://doi.org/10.1007/s00125002-0883-9)

Rezania A, Bruin JE, Arora P, Rubin A, Batushansky I, Asadi A, O’Dwyer S, Quiskamp N, Mojibian M, Albrecht T, et al. 2014 Reversal of diabetes with insulin-producing cells derived in vitro from human pluripotent stem cells. Nature Biotechnology 32 1121-1133. (https:// doi.org/10.1038/nbt.3033)

Rorsman P \& Renström E 2003 Insulin granule dynamics in pancreatic beta cells. Diabetologia 46 1029-1045. (https://doi.org/10.1007/ s00125-003-1153-1)

Rozzo A, Meneghel-Rozzo T, Delakorda SL, Yang SB \& Rupnik M 2009 Exocytosis of insulin: in vivo maturation of mouse endocrine pancreas. Annals of the New York Academy of Sciences 1152 53-62. (https://doi.org/10.1111/j.1749-6632.2008.04003.x)

Russ HA, Parent AV, Ringler JJ, Hennings TG, Nair GG, Shveygert M, Guo T, Puri S, Haataja L, Cirulli V, et al. 2015 Controlled induction of human pancreatic progenitors produces functional beta-like cells in vitro. EMBO Journal 34 1759-1772. (https://doi.org/10.15252/ embj.201591058)

Thompson PJ, Shah A, Ntranos V, van Gool F, Atkinson M, Bhushan A \& Gool F 2019 Targeted elimination of senescent beta cells prevents type 1 diabetes. Cell Metabolism 29 1045.e10-1060.e10. (https://doi. org/10.1016/j.cmet.2019.01.021)

van der Meulen T \& Huising MO 2014 Maturation of stem cell-derived beta-cells guided by the expression of urocortin 3. Review of Diabetic Studies 11 115-132. (https://doi.org/10.1900/RDS.2014.11.115)

van der Meulen T, Xie R, Kelly OG, Vale WW, Sander M \& Huising MO 2012 Urocortin 3 marks mature human primary and embryonic stem cell-derived pancreatic alpha and beta cells. PLOS ONE 7 e52181. (https://doi.org/10.1371/journal.pone.0052181)

van der Meulen T, Donaldson CJ, Cáceres E, Hunter AE, Cowing-Zitron C, Pound LD, Adams MW, Zembrzycki A, Grove KL \& Huising MO 2015 Urocortin3 mediates somatostatin-dependent negative feedback control of insulin secretion. Nature Medicine 21 769-776. (https://doi. org/10.1038/nm.3872)

van der Meulen T, Mawla AM, DiGruccio MR, Adams MW, Nies V, Dolleman S, Liu S, Ackermann AM, Cáceres E, Hunter AE, et al. 2017 Virgin beta cells persist throughout life at a neogenic niche within pancreatic islets. Cell Metabolism 25 911.e6-926.e6. (https://doi. org/10.1016/j.cmet.2017.03.017)

Velazco-Cruz L, Song J, Maxwell KG, Goedegebuure MM, Augsornworawat P, Hogrebe NJ \& Millman JR 2019 Acquisition of dynamic function in human stem cell-derived $\beta$ cells. Stem Cell Reports 12 351-365. (https://doi.org/10.1016/j. stemcr.2018.12.012)

Veres A, Faust AL, Bushnell HL, Engquist EN, Kenty JHR, Harb G, Poh YC, Sintov E, Gürtler M, Pagliuca FW, et al. 2019 Charting cellular identity during human in vitro $\beta$-cell differentiation. Nature 569 368-373. (https://doi.org/10.1038/s41586-019-1168-5)

Wicksteed B, Brissova M, Yan W, Opland DM, Plank JL, Reinert RB, Dickson LM, Tamarina NA, Philipson LH, Shostak A, et al. 2010 Conditional gene targeting in mouse pancreatic $\beta$-cells: analysis of ectopic cre transgene expression in the brain. Diabetes 59 3090-3098. (https://doi.org/10.2337/db10-0624)

Xie R, Everett LJ, Lim HW, Patel NA, Schug J, Kroon E, Kelly OG, Wang A, D'Amour KA, Robins AJ, et al. 2013 Dynamic chromatin remodeling mediated by polycomb proteins orchestrates pancreatic differentiation of human embryonic stem cells. Cell Stem Cell 12 224-237. (https://doi.org/10.1016/j.stem.2012.11.023)

Received in final form 28 March 2020

Accepted 4 May 2020

Accepted Manuscript published online 5 May 2020 https://joe.bioscientifica.com https://doi.org/10.1530/JOE-19-0535 (c) 2020 Society for Endocrinology Published by Bioscientifica Ltd.
Printed in Great Britain 